\title{
Aspectos reprodutivos de Palaemon pandaliformis (Stimpson) (Crustacea, Decapoda, Palaemonidae) no Rio Sahy, Mangaratiba, Rio de Janeiro, Brasil
}

\author{
Giovana Valverde Lima ${ }^{1}$ \\ Lídia Miyako Yoshii Oshiro ${ }^{1}$
}

\begin{abstract}
The reproductive aspects of the freshwater shrimp Palaemon pandaliformis (Stimpson) (Crustacea, Decapoda, Palaemonidae) from Sahy River, Mangaratiba, Rio de Janeiro, Brazil. The reproductive aspects of the freshwater shrimp Palaemon pandaliformis (Stimpson, 1871) were studied from March, 1998 to February, 1999. Monthly, samples were collected with sives, on the river edge, under the edging vegetation or in small pools. At the laboratory, shrimps were sexed and measured in their total lenght, from the tip of the rostrum to the of telson with precision callipters. The ovigerous shrimps were separated and counted for fecundity determination. The eggs diameter was measured with calibrated stereomicroscope. A total of 872 were collected among which, $46 \%$ female, $29 \%$ male and $25 \%$ juvenil. The sex ratio was 1:1.57. Ovigerous females were present during all seasons, but the reproductive peak accurred in Spring. The ovigerous female showed total lenght from 23.0 to $39.6 \mathrm{~mm}$. The mean fecundity was 137 eggs per female, with a minimum of 40 eggs and a maximum of 270 eggs. The first sexual maturation probably occur at $23.0 \mathrm{~mm}$ total lenght. The mean diameter os the eggs size was $0.51 \mathrm{~mm}$ (minor) and $0.73 \mathrm{~mm}$ (major)
\end{abstract}

KEY WORDS. Reproduction, Palaemon pandaliformis, freshwater shrimp, Sahy River, Rio de Janeiro State

Palaemon pandaliformis (Stimpson, 1871) é uma espécie de camarão de pequeno porte, que ocorre nas regiões tropicais, subtropicais e temperadas de todo o mundo (Holthuis 1980). De acordo com Ramos-PoRTo \& CoElHo (1998), esta espécie no Brasil ocorre do Rio Grande do Norte ao Rio Grande do Sul.

Segundo BOND-BUCKUP \& BUCKUP (1989), P. pandaliformis é a única espécie de água doce do gênero que ocorre nas bacias do Brasil Meridional, sendo encontrada com frequiência junto às raízes de vegetação aquática. No nordeste brasileiro, a espécie é conhecida popularmente como potitinga (HOLTHUIS 1980).

São poucos os estudos sobre a espécie, principalmente no que se refere à biologia reprodutiva. COELHO (1963) e MüLLER et al. (1996, 1999), estudaram os aspectos reprodutivos da espécie, respectivamente nos Estados de Pernambuco e Santa Catarina.

Este trabalho procurou ampliar as informações sobre a espécie e contribuir para o conhecimento de sua biologia reprodutiva no Rio Sahy, Mangaratiba, Estado do Rio de Janeiro.

1) Estação de Biologia Marinha, Universidade Federal Rural do Rio de Janeiro. Rua Sereder, Itacuruçá, 23860-020 Mangaratiba, Rio de Janeiro. E-mail: oshiro@ufrrj.br 


\section{MATERIAL E MÉTODOS}

Palaemon pandaliformis foi coletado mensalmente no período de março/1998 a fevereiro/1999 no Rio Sahy, Município de Mangaratiba, litoral sul do Estado do Rio de Janeiro (22 $56^{\prime}$ 'S; $44^{\circ} 01^{\prime} \mathrm{W}$ ).

A área de estudo está localizada numa região estuarina, com substrato arenolodoso. Foram estabelecidas duas estações de acordo com a distância em relação à foz. A Estação I se caracteriza por receber menor influência da salinidade; enquanto a Estação II, apresenta maior influência da salinidade por estar mais próxima à foz.

Todos os exemplares foram capturados através de peneiras com malha de $1,0 \mathrm{~mm}$, passados sob a vegetação marginal e substratos depositados sobre o fundo.

Foram registrados dados ambientais em relação à temperatura do ar e da água, salinidade e a profundidade dos locais de coleta.

Os camarões coletados foram levados ao laboratório da Estação de Biologia Marinha, Universidade Federal Rural do Rio de Janeiro (UFRRJ) em Itacuruçá, Rio de Janeiro, fixados em formaldeído $10 \%$, posteriormente conservados em álcool a $70 \%$ e etiquetados.

Cada indivíduo foi medido com paquímetro tomando-se o comprimento total (medida compreendida entre a margem anterior do rostro e a porção distal do telso) e o comprimento do cefalotórax (medida da margem posterior do pedúnculo ocular até a margem distal do cefalotórax).

A razão sexual populacional foi determinada através do número total de fêmeas e machos capturados durante os meses de coletas.

O período reprodutivo foi estabelecido com base na frequiência relativa de fêmeas ovígeras ao longo das estações do ano.

O tamanho da maturidade sexual individual foi determinada pelo comprimento apresentado pela menor fêmea ovígera capturada, enquanto que a maturidade sexual populacional foi estimada para a classe de comprimento total em que se encontram $50 \%$ do número de fêmeas ovígeras da população (VAZZOLER 1981).

Para o estudo da fecundidade foram utilizadas 50 fêmeas ovígeras, obtidas aleatoriamente das amostras mensais. Os ovos foram retirados da câmara incubadora e separados com auxílio de pinças e estiletes, sendo contados em sua totalidade com contador manual sob um microscópio estereoscópico $25 \mathrm{x}$.

A relação entre o número de ovos e o comprimento do cefalotórax foi estimada pela equação $\mathrm{Y}=\mathrm{a}+\mathrm{bX}$.

A medição dos ovos foi feita através de uma amostra aleatória de 20 ovos de cada fêmea, tomando-se o comprimento e a largura, utilizando-se uma ocular micrométrica acoplada ao microscópio estereoscópico.

\section{RESULTADOS \& DISCUSSÃO}

A temperatura média do ar nos locais de coleta foi de $25 \pm 5,0^{\circ} \mathrm{C}$; enquanto a temperatura média da água foi de $23 \pm 4,0^{\circ} \mathrm{C}$. Os valores da temperatura tanto do ar quanto da água, apresentaram os menores e maiores valores nos meses de junho e janeiro, respectivamente. A salinidade no Rio Sahy variou de 0 a $5 \%$ e a profundidade não ultrapassou $50 \mathrm{~cm}$, sendo a média anual em torno de $42 \mathrm{~cm}$. 
Foram capturados um total de 872 indivíduos dos quais, 46\% fêmeas, $29 \%$ machos e $25 \%$ juvenis. Do total de fêmeas amostradas, $17,4 \%$ eram formas ovígeras. A razão sexual obtida para a população foi de 1:1,57 observando-se uma predominância de fêmeas na população.

De acordo com WILLSON \& PIANKA (1963), a proporção entre o número de machos e fêmeas pode diferir devido a diversos fatores, os quais podem afetar mais um sexo que outro.

Segundo CHRISTY \& SALMON (1984), a proporção sexual desviada para as fêmeas pode estar relacionada ao sistema de acasalamento das espécies.

As fêmeas apresentaram comprimento total que variou de 16,1 a $40,0 \mathrm{~mm}$ (3,0 a $8,0 \mathrm{~mm}$ de comprimento do cefalotórax), tamanho um pouco inferior em relação à população de Santa Catarina, que variou de 16,0 a 48,0 mm (MülLER et al. 1996). Os machos estudados apresentaram tamanhos entre 14,1 a $42,0 \mathrm{~mm}(4,0$ a $6,4 \mathrm{~mm}$ de comprimento do cefalotórax), diferindo em relação aos estudos anteriores: MÜLLER et al. (1996), de 20,0 a 42,0 mm, e BOND-BUCKUP \& BUCKUP (1989) de 22,0 a $30,0 \mathrm{~mm}$. As fêmeas ovígeras apresentaram comprimento total variando entre 23,0 a $39,6 \mathrm{~mm}$ ( 5,2 a 6,7 mm de comprimento do cefalotórax). Estes valores são inferiores aos apresentados por MÜLLER et al. (1996), que observaram tamanhos entre 27,4 a $50,4 \mathrm{~mm}$ de comprimento total. Os menores tamanhos observados para a região do Rio Sahy, provavelmente está relacionada com as variações fotoperiódicas que são menos pronunciadas nas regiões equatoriais e nas baixas latitudes (BOND \& BUCKUP 1982).

As fêmeas ovígeras foram encontradas durante o ano todo, apresentando um pico reprodutivo durante a primavera (Fig. 1). Todos os indivíduos coletados foram capturados sempre sob a vegetação marginal, concordando com as observações de (MÜLlER et al. 1996).

Segundo SANTOS (1978), o período reprodutivo pode ser contínuo, se as fêmeas desovam com a mesma intensidade durante o ano todo, ou periódico ou descontínuo, se for verificado exclusivamente, ou com maior intensidade em determinadas épocas do ano. A espécie estudada apresenta uma reprodução do tipo periódica, uma vez que a reprodução ocorreu com maior intensidade durante uma estação do ano, reduzindo suas freqüências no decorrer das demais, provavelmente para recuperação gonadal após intenso período reprodutivo.

A maturidade sexual individual de $P$. pandaliformis foi verificada com 23,0 $\mathrm{mm}$ de comprimento total (5,4 $\mathrm{mm}$ de comprimento do cefalotórax), enquanto a maturidade sexual populacional estimada para a população foi encontrada na classe de tamanho de 28,1-32,0 mm de comprimento total (5,8-7,0 $\mathrm{mm}$ de comprimento do cefalotórax) (Fig. 2). No Manguezal de Ratones, Florianópolis, Santa Catarina, a maturidade sexual individual foi de $27,0 \mathrm{~mm}$, enquanto a maturidade sexual populacional foi estimada na classe de tamanho entre 36,0-41,0 mm (MüLLER et al. 1996). A população do presente estudo, apresentou uma maturidade sexual precoce, provavelmente devido às diferenças de latitudes entre as duas áreas de estudo.

De acordo com WENNER et al. (1985), as variações no tamanho da maturidade são comuns em crustáceos podendo ser causadas por variações na taxa de crescimento e por vários graus de variabilidade que podem ocorrer sazonalmente ou em diferentes localidades. 

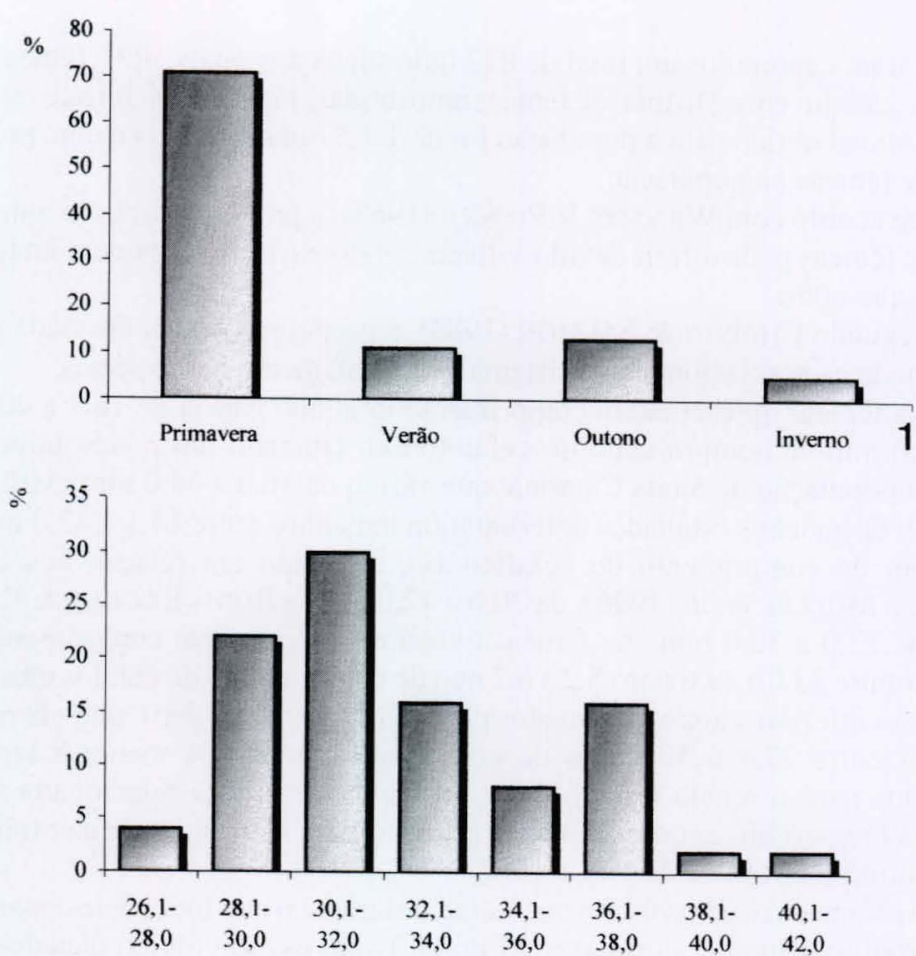

Classes de comprimento total ( $\mathrm{mm})$

2

Figs 1-2. Fêmeas ovigeras de Palaemon pandaliformis no Rio Sahy, durante o período de março/1998 a fevereiro/1999: (1) distribuição sazonal; (2) freqüência relativa de acordo com as classes de comprimento.

Os ovos apresentaram forma elíptica e tamanho que variou de 0,65 a 0,84 mm de comprimento, e 0,46 a $0,57 \mathrm{~mm}$ de largura. $\mathrm{O}$ tamanho médio observado foi de 0,73 e $0,54 \mathrm{~mm}(0,28 \pm 0,22)$, respectivamente, em comprimento e largura. No entanto, não foi possível a observação do aumento de tamanho em relação ao desenvolvimento embrionário, uma vez que as fêmeas ovígeras capturadas encontravam-se todas em início de postura.

A fecundidade média para a espécie variou de 40 a 270 ovos, obtendo-se um valor médio de $137 \pm 48,4$ ovos. A maior freqüência de fêmeas ovígeras foi encontrada na classe de 106 a 139 ovos (Fig. 3). Os valores encontrados para a espécie são inferiores aos registrados na região sul do Brasil, onde a variação foi de 107 a 684 ovos, com média aproximada de 420 ovos (MÜLLER et al. 1996). Essa diferença pode ser explicada através do pequeno porte apresentado pelas fêmeas ovígeras ou devido às atividades mecânicas durante o processo de coleta.

De acordo com OH \& HaRTNOLl (1999), as condições fisiológicas das fêmeas, estação do ano, latitude e condições ambientais propiciam diferenças em relação ao número de ovos. Segundo AmMAR et al. (2001), as variações na 
estimativa da fecundidade podem ser decorrentes de diferenças no tamanho máximo dos exemplares e das características genéticas próprias das populações de origem.

A equação que descreve a relação entre o número de ovos carregados pelas fêmeas ovígeras e o comprimento do cefalotórax foi $\mathrm{Y}=28,83 \mathrm{x}-45,062\left(\mathrm{r}^{2}=\right.$ 0,2109) (Fig. 4). Verificando-se que o número de ovos, da espécie em estudo, aumenta em função do aumento da largura da carapaça, como observado em outros palaemonídeos.
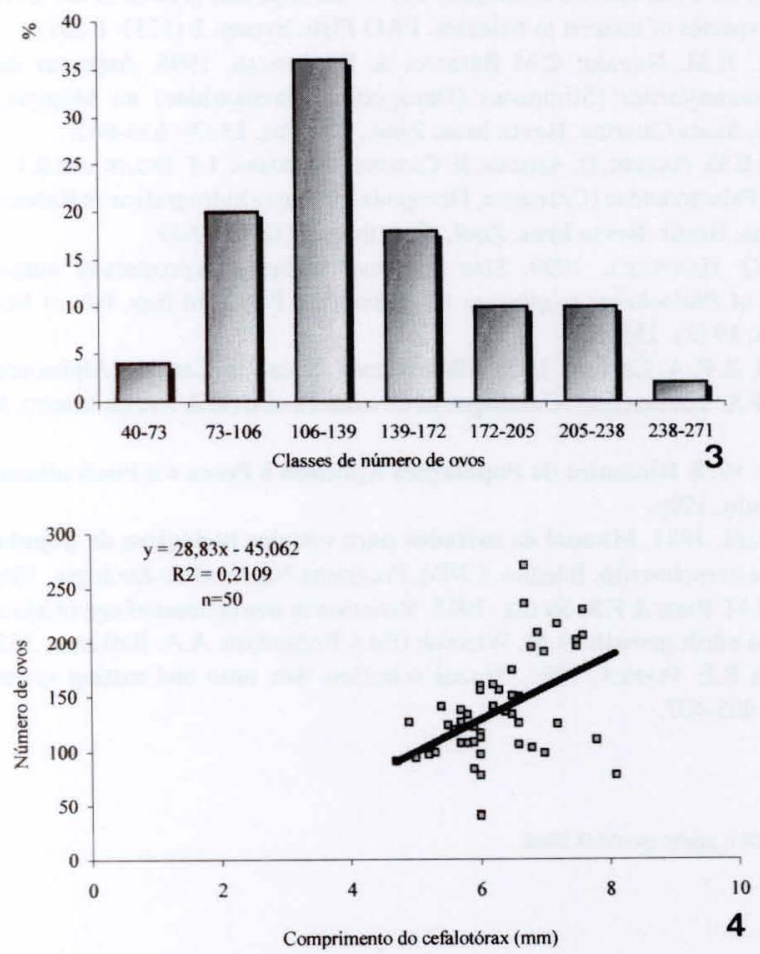

Figs 3-4. Palaemon pandaliformis no Rio Sahy, durante o periodo de março/1998 a fevereiro/1999: (3) freqüência relativa de fêmeas ovígeras de acordo com as classes de número de ovos; (4) distribuição do número de ovos de acordo com o comprimento do cefalotórax ( $\mathrm{mm}$ ).

AGRADECIMENTOS. Ao Sr. Casemiro Antônio Alves e ao Sr. Benedito Albernaz Lima pelo auxílio nas coletas mensais realizadas.

\section{REFERÊNCIAS BIBLIOGRÁFICAS}

Ammar, D.; Y. M.R. Müller \& E.M. NAZARI. 2001. Biologia reprodutiva de Macrobrachium olfersii (Wiegmann) (Crustacea, Decapoda, Palaemonidae) coletados na Ilha de Santa Catarina, Brasil. Revta bras. Zool., Curitiba, 18 (2): 529-537.

Bond, G. \& L. Buckup. 1982. O ciclo reprodutor de Macrobrachium borellii (Nobili, 1896) e Macrobrachium potiuna (Muller, 1880) (Crustacea, Decapoda, Palaemonidae) e suas relações com 
a temperatura. Rev. Brasil. Biol., Rio de Janeiro, 42 (3): 473-483.

Bond-Buckup, G. \& L. Buckup. 1989. Os Palaemonidae de águas continentais do Brasil Meridional

(Crustacea, Decapoda). Rev. Brasil. Biol., Rio de Janeiro, 49 (4): 883-896.

Christy, J.H. \& M. SALmon. 1984. Ecology and evolution of mating systems of fiddler crabs (Genus Uca). Biol. Rev., Cambridge, 59: 483-509.

CoElHo, P.A. 1963. Algumas observações sobre a biologia e ecologia dos camarões Palaemon northropi e P. pandaliformis no Estado de Pernambuco (Decapoda, Palaemonidae). Bol. Inst. Oceanogr., São Paulo, 5/6: 69-72.

Holthuis, L.B. 1980. FAO species catalogue. Vol. 1. Shrimps and prawns of the world. Na annotated catalogue of species of interest to fisheries. FAO Fish. Synop. 1 (125): 1-261.

Müller, Y.M.R.; E.M. NazAri; C.M Bressan \& D. Ammar. 1996. Aspectos da reprodução de Palaemon pandaliformis (Stimpson) (Decapoda, Palaemonidae) no Manguezal de Ratones, Florianópolis, Santa Catarina. Revta bras. Zool., Curitiba, 13 (3): 633-642.

MÜller, Y.M.R.; E.M. NAZARI; D. Ammar; E. CARgnin-Ferreira; I.T. Beltrame \& C. PACheCo. 1999. Biologia dos Palaemonidae (Crustacea, Decapoda) da Bacia hidrográfica de Ratones, Florianópolis, Santa Catarina, Brasil. Revta bras. Zool., Curitiba, 16 (3): 629-639.

OH, C.W. \& R.G. HARTNOLl. 1999. Size at sexual maturity, reproductive output, and seasonal reproduction of Philocheras trispinosus (Decapoda) in Port Erin Bay, Isle of Man. Jour. Crust. Biol., Florida, 19 (2): 252-259.

Ramos-Porto, M. \& P. A. Coelho. 1998. Malacostraca. Eucarida. Caridae (Alpheoidea exclueded), p. 325-350. In: P.S. Young (Ed.). Catalogue of Crustacea of Brazil. Rio de Janeiro, Museu Nacional, $717 \mathrm{p}$.

Santos, E.P. Dos. 1978. Dinâmica de Populações Aplicada à Pesca e a Piscicultura. São Paulo, Ed. Univ. São Paulo, 129p.

VAZZoler, A.E.A.M. 1981. Manual de métodos para estudos biológicos de populações de peixes: reprodução e crescimento. Brasília, CNPq, Programa Nacional de Zoologia, 106p.

Wenner, A.M.; H.M. PAGE \& P.R. Siegel. 1985. Variation in size at onset of egg production, p. 149-163. $I n$ : Factors in adult growth. A.M. WenNer (Ed.). Rotterdam, A.A. Balkema, 362p.

Willson, M.F. \& R.E. Pianka. 1963. Sexual selection, Sex ratio and mating system. Amer. Nat., Chicago, 97: 405-407.

Recebido em 14.IX.2001; aceito em 03.IX.2002. 